\title{
Bacillary angiomatosis compatible with systemic manifestations: a case report
}

\author{
Monica Rueda ${ }^{1,2}$, Natalie Torres ${ }^{1,2}$, Alex Ventura ${ }^{3}$ \\ ${ }^{1}$ Hospital Nacional Dos de Mayo, Lima, Peru \\ ${ }^{2}$ Universidad Nacional Mayor de San Marcos, Lima, Peru \\ ${ }^{3}$ Hospital Nacional Cayetano Heredia, Lima, Peru
}

\begin{abstract}
Bacillary angiomatosis is a rare infection caused by Bartonella henselae or Bartonella quintana, which usually affects patients with the human immunodeficiency virus (HIV) and causes cutaneous and extracutaneous lesions; it is usually benign, but potentially fatal due to systemic involvement. A 49-yearold male, HIV-positive patient was admitted for fever, weight loss, respiratory distress, lymphadenopathy, edema, and multiple widespread angiomatous papules, which began 2 months prior to admission. He had pancytopenia, hypoalbuminemia and hepatosplenomegaly. Skin biopsy showed a well-demarcated nodular lesion with an epidermal collaret that enclosed the dermis, and proliferation of capillaries with hyperplasia and hypertrophy of the endothelial cells. Bacilli were observed with Warthin-Starry stain. He was treated with cotrimoxazole, obtaining resolution of skin lesions and other systemic disorders; however, after stopping therapy, he was readmitted because of recurrence, then he died. This case shows a clinical-pathological correlation of bacillary angiomatosis, which was confirmed by Warthin-Starry stain. In addition, it was associated with systemic findings supported by the therapeutic response to antibiotics, the recurrence of the disease and the exclusion of other differential diagnoses. Despite the reduction of opportunist infections, this unusual disease could be present in some patients and affect skin and other tissues, which increases the probability of death, so physicians should recognize it and start therapy appropriately.
\end{abstract}

HIV AIDS Rev 2019; 18, 1: 74-77

DOI: https://doi.org/10.5114/hivar.2019.83818

Key words: HIV, bacillary angiomatosis, peliosis hepatis, Bartonella.

\section{Introduction}

Bacillary angiomatosis is a rare infectious disease caused by a gram-negative bacillus from the genus Bartonella (B. henselae and B. quintana), which is often described in patients infected with the human immunodeficiency virus (HIV). It is included within category B according to the Centers for Disease Control and Prevention (CDC), although it has been observed in patients with CD4 counts less than 200. The prevalence is unknown. Some studies report an in-

Address for correspondence: Dr. Natalie Torres, Hospital Nacional Dos de Mayo, Avenida Miguel Grau No 1300 , 15072, Lima, Peru, phone: (+511)997089133,

e-mail: torrespnat.14@gmail.com

cidence of 1.2 and 1.42 per 1,000 HIV patients; however, it is considered underdiagnosed $[1,2]$. Cats are the main reservoir for $B$. henselae, then transmission to humans can occur via a bite, scratch, or cat fleas. Humans are considered the reservoir for B. quintana, which is transmitted through body lice [3]. The bacteria stimulate endothelial cell proliferation, which manifests as vascular tumors in different tissues [4]. It appears as single or multiple angiomatous papules or nodules on the skin and can involve the liver, spleen, and bone [5]. Although the course is usually benign, it is potentially fatal

Article history:

Received: 22.04.2017

Received in revised form: 01.12.2018

Accepted: 03.12.2018

Available online: 10.03.2019
International Journal of HIV-Related Problems

HIV \& AIDS

R e vi e w 
$[1,3]$. We report a case of an immunosuppressed patient with bacillary angiomatosis compatible with systemic manifestations.

\section{Case report}

A 49-year-old man from Lima (central coast of Peru) with HIV infection without antiretroviral treatment developed fever, weight loss, dyspnea, lymphadenopathy, edema, and generalized papular lesions on his skin 2 months before examination. He recalled pulmonary and lymph node multidrug-resistant tuberculosis fully treated 2 years before admission and illicit drug abuse. Contact with cats was recorded.

At admission time (Hospital Nacional Dos de Mayo), hypoxemia was found. We observed multiple and well-defined angiomatous papules scattered on the face, trunk, and extremities (red-purple colored and from 1 to $3 \mathrm{~mm}$ in diameter) (Figures 1 and 2), and a well-defined purple nodule in the right lateral cervical region of $8 \mathrm{~mm}$ in diameter (Figure 3). Edema of lower limbs and cervical lymphadenopathy were found.

As part of the laboratory tests, pancytopenia and hypoalbuminemia were highlighted. The CD4 count was 42 cell/ $\mathrm{mm}^{3}$. Sputum, urine, and feces smears for tuberculosis, and serologic tests for syphilis and toxoplasmosis, other agents, rubella, cytomegalovirus, and herpes simplex $(\mathrm{TORCH})$ were negative.

Chest X-ray showed a bilateral interstitial pattern, and computed tomography (CT) (performed 14 days after starting treatment) revealed diffuse hepatosplenomegaly and reactive mediastinal lymphadenopathy.

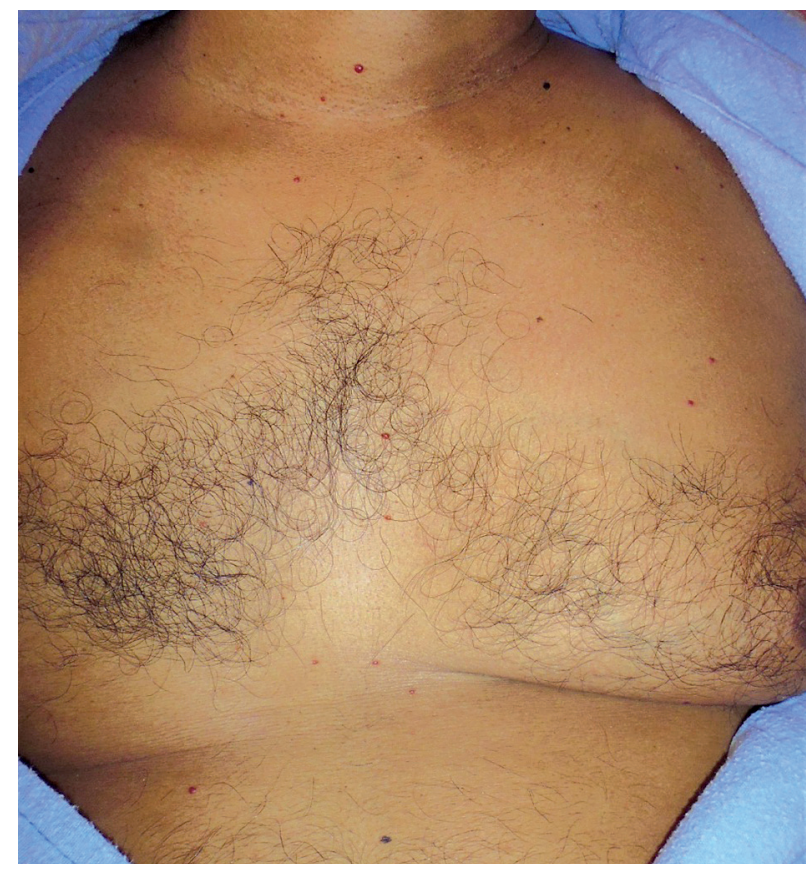

Figure 2. Multiple red-purple scattered on the trunk
Skin biopsy showed a well-demarcated nodular lesion with an epidermal collaret that enclosed the dermis (Figure 4), and the most important finding was the proliferation of capillaries with hyperplasia and plump of the endothelial cells. A histiocytic reaction and chronic inflammation were also observed, and in some areas, we observed the presence of eosinophilic material, which was slightly blurred (Figures 4 and 5). These findings were

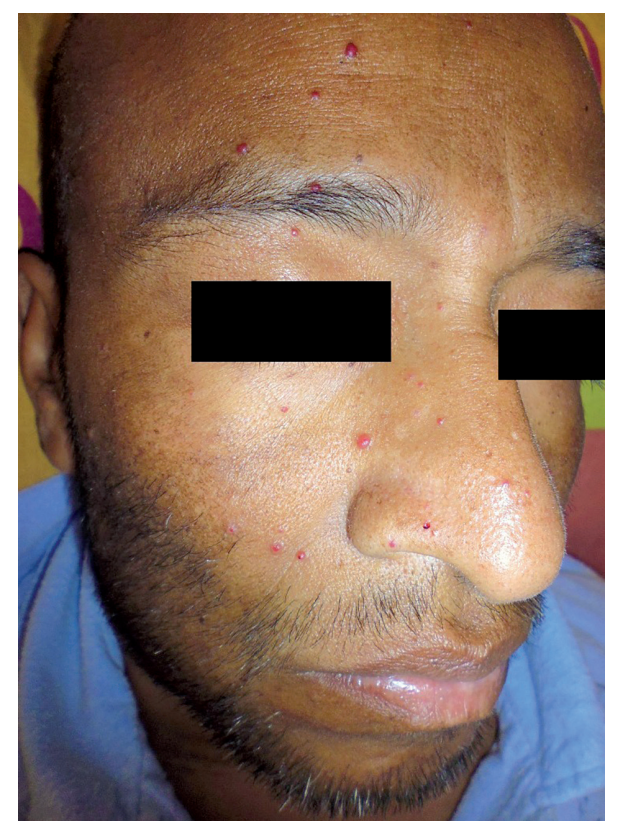

Figure 1. Multiple red-purple, 1 to $3 \mathrm{~mm}$ in diameter, well defined on the face

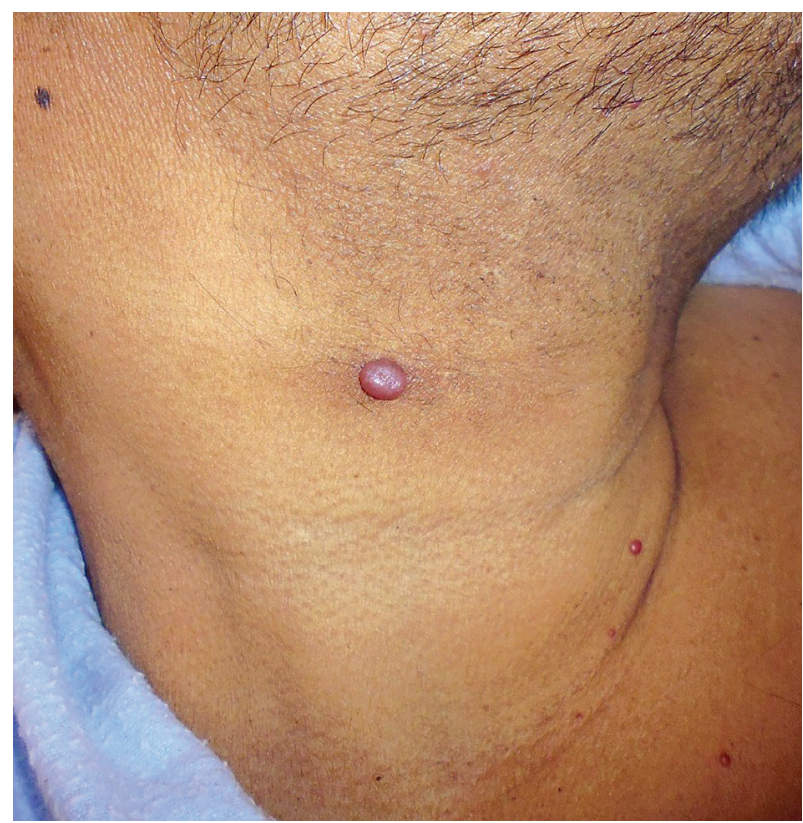

Figure 3. Purple nodule of $8 \mathrm{~mm}$ in diameter on right lateral cervical region 


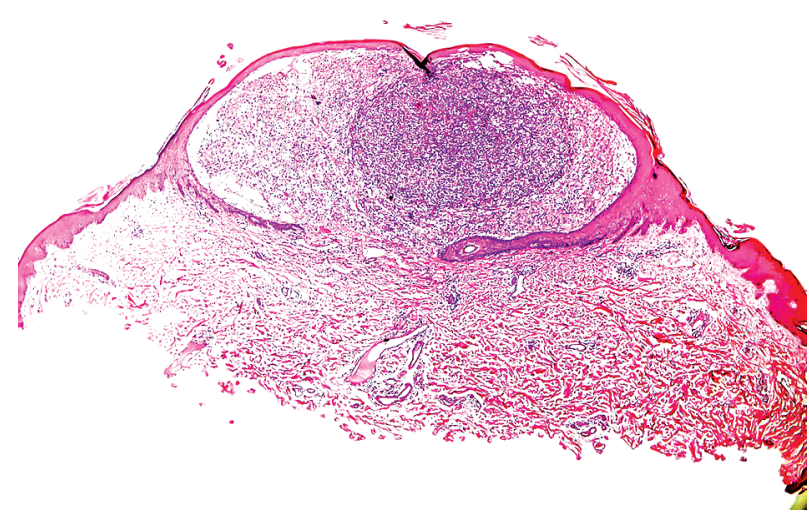

Figure 4. Nodular lesion with an epidermal collaret that enclosed the dermis (HE, 4x)

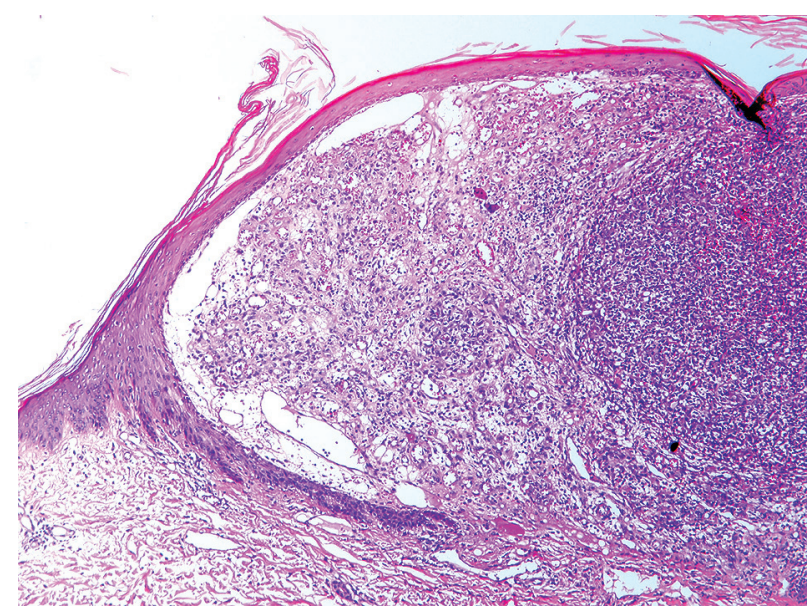

Figure 5. Proliferation of capillaries with hyperplasia and plump of the endothelial cells and areas with eosinophilic material (HE, 20x)

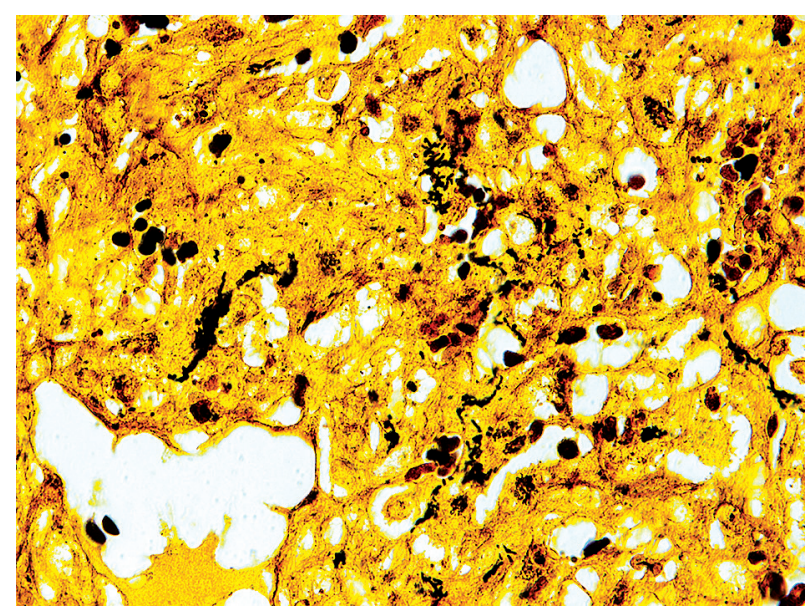

Figure 6. Clusters of bacillary structures (Warthin-Starry stain, 100x)

highly suspicious for bacillary angiomatosis (according to the clinical context). A Warthin-Starry stain was performed, and we found the presence of bacillary structures, some in clusters and others isolated (Figure 6). The study of the cervical lymph node showed non-specific lymphoid hyperplasia.

Ventilatory improvement and remission of fever and skin lesions were observed in the second week after treatment with cotrimoxazole because of Pneumocystis pneumonia, but without hematological changes. However, the patient applied for voluntary discharge in a stable condition, and then he dropped out of treatment. After 1 month, he was readmitted in poor general condition because of respiratory failure, liver failure, severe pancytopenia, and reappearance of cutaneous lesions. Clinical evolution was rapid, and then he died due to septic shock.

\section{Conclusions}

Bacillary angiomatosis can affect different tissues and produce vascular tumors secondary to angiogenesis. The most common manifestations are reported in skin as polymorphic lesions: papules or nodules, red, purple or skin colored, ranging in size from a few millimeters to several centimeters, compressible, tense or friable, with smooth, warty or ulcerated surface, localized or disseminated. Fever, weight loss, and hyporexia may accompany or precede the onset of the outbreak $[1,5]$. All these clinical findings were found in our patient, being characteristic of bacillary angiomatosis.

Bacillary angiomatosis can be invasive and affect tissues such as the lymph nodes, liver and spleen, with or without cutaneous lesions [6]. Bone marrow and the respiratory system have also been reported as affected [5]. Splenic or liver involvement (bacillary peliosis) is formed by bleeding cavities from $0.5 \mathrm{~mm}$ to several centimeters that are evidenced by ultrasound or CT, although this does not detect cysts less than $1 \mathrm{~cm}$ [7]. The clinical course is variable, ranging from resolution with treatment to acute liver failure. Often hepatosplenomegaly is found, and it can be accompanied by lymphadenopathy and anemia [6]. The size of cysts and the time at which tomography was performed may have made the observation of the lesions difficult in this case. However, other causes of hepatosplenomegaly were ruled out.

In clinical practice, biopsy is recommended to confirm the disease when cutaneous lesions are present. WarthinStarry stain allows visualization of clumps of bacilli and confirms the histological diagnosis. Serology (immunofluorescence assays detecting immunoglobulin $\mathrm{G}$ [ $\mathrm{IgG}]$ and immune enzyme assays) is a useful and sensitive method. Nevertheless, detection of Bartonella species can be made through molecular or culture isolation. Culture has a low sensitivity. In contrast, polymerase chain reaction (PCR) is recommended and considered more sensitive than the former $[3,8]$.

The main differential diagnosis in immunosuppressed patients with proliferative vascular lesions includes Kaposi's sarcoma. Other diagnoses to consider are pyogenic granuloma, hemangiomas, and cutaneous lymphomas $[8,9]$.

Development of pancytopenia suggests bone marrow involvement. It has been attributed to hypersplenism and bone marrow infiltration in previous cases reported in the litera- 
ture $[1,10]$. Unfortunately, liver and bone marrow samples could not be collected to demonstrate the presence of bacilli.

The treatment of choice is erythromycin $500 \mathrm{mg}$ qid and doxycycline $100 \mathrm{mg}$ bid for 2 to 8 weeks. Other antibiotics such as aminoglycosides, quinolones, cotrimoxazole, and some cephalosporins show activity against this bacillus [11]. The favorable therapeutic response to antibiotics and the recurrence of the disease after leaving treatment suggest an infectious etiology consistent with bacillary angiomatosis. However, it was not possible to identify a Bartonella species.

Despite the remarkable reduction of opportunistic infections in the antiretroviral era, physicians should regard bacillary angiomatosis as a possible diagnosis in HIV patients and consider its cutaneous and extracutaneous findings in order to make an early diagnosis and provide appropriate and timely management. Otherwise, it can contribute to mortality in these patients.

\section{Conflict of interest}

The authors declare no potential conflicts of interest with respect to the research, authorship, and/or publication of this article.

\section{References}

1. Gazineo JL, Trope BM, Maceira JP, et al. Bacillary angiomatosis: description of 13 cases reported in five reference centers for AIDS treatment in Rio de Janeiro, Brazil. Rev Inst Med Trop Sao Paulo $2001 ; 43: 1-6$.

2. Plettenberg A, Lorenzen T, Burtsche BT, et al. Bacillary angiomatosis in HIV-infected patients - an epidemiological and clinical study. Dermatology 2000; 201: 326-331.

3. Akram SM, Bhimji SS. Angiomatosis, bacillary. StatPearls Publishing, Treasure Island 2018.

4. Dehio C. Bartonella-host-cell interactions and vascular tumour formation. Nat Rev Microbiol 2005; 3: 621-631.

5. Velho PE, Souza EM, Cintra ML, et al. Angiomatose bacilar: revisão da literatura e documentação iconográfica. An Bras Dermatol 2003; 78: 601-609.

6. Mohle-Boetani JC, Koehler JE, Berger TG, et al. Bacillary angiomatosis and bacillary peliosis in patients infected with human immunodeficiency virus: clinical characteristics in a case-control study. Clin Infect Dis 1996; 22: 794-800.

7. Crocetti D, Palmieri A, Pedullà G, et al. Peliosis hepatis: personal experience and literature review. World J Gastroenterol 2015; 21: 13188-13194.

8. Uribe P, Balcells ME, Giesen L, et al. Angiomatosis bacilar por Bartonella quintana como primera manifestación de infección por VIH. Rev Med Chile 2012; 140: 910-914.

9. Eyer-Silva WA, Soares PE, Azevedo MC, et al. An unusual case of bacillary angiomatosis in the oral cavity of an AIDS patient who had no concomitant tegumentary lesions - case report and review. Rev Inst Med Trop Sao Paulo 2017; 59: e59.

10. Velho PE, Pimentel V, Del Negro GM, et al. Severe anemia, panserositis, and cryptogenic hepatitis in an HIV patient infected with Bartonella henselae. Ultrastruct Pathol 2007; 31: 373-377.

11. Stevens DL, Bisno AL, Chambers HF, et al. Practice guidelines for the diagnosis and management of skin and soft tissue infections: 2014 update by the Infectious Diseases Society of America. Clin Infect Dis 2014; 59: e10-52. 